\title{
COVID-19 vaccine roll-out in South Africa: The added value of wastewater surveillance for SARS-CoV-2
}

There has been unprecedented progress in vaccine development against SARS-CoV-2, the virus that causes COVID-19. However, given the huge demand for a limited supply of efficacious vaccines, there has been global, regional and local outcry regarding the lack of access to vaccines and the equity of distribution. Over 319 million vaccine doses have been administered (at the time of writing), with the majority in high-income countries. ${ }^{[1,2]}$ In contrast, by mid-January 2021, Guinea was the sole low-income country to have administered vaccines, but to only 25 individuals. ${ }^{[3]}$ Early in February 2021, South Africa (SA) received one million doses of the AstraZeneca vaccine; however, shortly thereafter, data reflected disappointing results on the efficacy of this vaccine on the predominant strain in the country (the 501Y.V2 variant), prompting the country to reverse the decision to utilise these in the national roll-out. With ample agility, on 17 February 2021 SA launched an early-access programme through a phase 3B open-label study using the Johnson \& Johnson vaccine candidate, rapidly deploying the first tranche of 80000 vaccines within hours of the vaccines touching down on SA soil.

Despite global surveillance efforts and the promise of numerous vaccine candidates, ongoing public health challenges to fully understand the impact of the COVID-19 pandemic remain a major concern. COVID-19 testing data have become a key metric that informs public health decision-making. However, epidemiological indicators can be flawed, as they may be influenced by insufficient testing capacity, limited access to healthcare facilities and testing bias, as individuals with severe symptoms are more likely to be tested than asymptomatic individuals. ${ }^{[4,5]}$ While African countries have reported low COVID-19 case numbers relative to other regions, this may not be indicative of low prevalence or spread containment, but rather low surveillance. ${ }^{[6]}$

\section{Wastewater surveillance as an early warning system}

Wastewater-based epidemiology has played an important role in the development of early warning systems for various enteric viruses, including poliovirus, norovirus and hepatitis. The detection of SARS-CoV-2 RNA in faeces and urine stimulated rapid research into wastewater surveillance for SARS-CoV-2. A wastewater sample collected from a wastewater treatment plant can represent the point prevalence of disease within the catchment community. Wastewater surveillance for SARS-CoV-2 is now carried out in over 50 countries ${ }^{[1,7]}$ to screen for disease presence in populations, provide an early warning for detection of COVID-19 cases in the community, monitor trends and track hot spots. ${ }^{[8]}$

In SA, a key milestone was both confirming the presence of SARSCoV-2 viral RNA (qualitative analysis) and determining the RNA copy number of SARS-CoV-2 viral RNA by reverse transcription polymerase chain reaction (quantitative analysis). ${ }^{[9]}$ The South African Medical Research Council's SARS-CoV-2 wastewater research and surveillance programme has set up wastewater monitoring in four provinces, namely Eastern Cape, Western Cape, Limpopo and Gauteng. The monitoring currently covers over 45 wastewater treatment plants with a total catchment population of $\sim 4$ million inhabitants. Results of the SARS-CoV-2 RNA signal are uploaded weekly on a dashboard (https://www.samrc.ac.za/wbe/) freely accessible to the public. Additional sampling sites are being added as the programme aims to expand surveillance to enable monitoring of hotspots, variants and other emerging pathogens.

\section{Can wastewater surveillance detect a COVID-19 vaccine-induced break in transmission?}

An efficacious COVID-19 vaccine, to prevent infection, disease or transmission, is essential for controlling the pandemic. ${ }^{[10]}$ Wastewater surveillance has the potential for use as a public health tool for guiding and monitoring national vaccine efforts. ${ }^{[11]}$ Firstly, in order to measure vaccine efficacy in real-world settings, there is a reliance on ongoing transmission of SARS-CoV-2, which is contradictory to public health promotion efforts. ${ }^{[10]}$ The level of ongoing community transmission can be monitored in wastewater, which in turn may provide insights into the impact of the vaccination programmes. Secondly, there are currently limited data on the efficacy of the current generation of vaccines to protect against COVID-19 variants. Wastewater monitoring may provide evidence on the SARS-CoV-2 RNA viral load and viral shedding trends during the roll-out of vaccinations in communities. Thirdly, for vaccinated, unvaccinated or mixed populations, wastewater surveillance can provide an early warning system for prioritising hotspots where vaccination coverage is most urgently required. And lastly, targeted and untargeted sequencing of wastewater pathogens has the potential to track the spread of specific sequence variants and identify mutations for which vaccinations may be need be modified. ${ }^{[12]}$

In conclusion, wastewater surveillance is currently being expanded in SA with a view to early detection of the imminent third wave of COVID-19. Recognising the shortage of resources and capacity to counteract COVID-19 in the country, including limited numbers of vaccines available, the expanded wastewater surveillance and early warning system could also be applied as a potentially efficient and cost-effective mechanism to evaluate the efficacy of the vaccination roll-out and identify locations where COVID-19 transmission remains intractable. It also holds the promise of supporting the long-term goal of monitoring COVID-19 disease absence in communities.

Acknowledgements. The South African Medical Research Council is thanked for funding and support.

Author contributions. All authors substantially contributed to the conception and design of the article and interpreting the relevant literature.

\section{Renée A Street, Angela Mathee}

Environment and Health Research Unit, South African Medical Research Council, Johannesburg, South Africa; and Environmental Health Department, Faculty of Health Sciences, University of Johannesburg, South Africa

renee.street@mrc.ac.za

\section{Rabia Johnson, Christo J F Muller}

Biomedical Research and Innovation Platform, South African Medical Research Council, Cape Town, South Africa; and Division of Medical Physiology, Faculty of Health Sciences, Stellenbosch University,

Cape Town, South Africa

\section{Johan Louw}

Biomedical Research and Innovation Platform, South African Medical Research Council, Cape Town, South Africa 


\section{Mongezi Mdhluli}

Chief Research Operations Office, South African Medical Research Council, Cape Town, South Africa

\section{Huub C Gelderblom}

Fred Hutchinson Cancer Research Center, Seattle, Wash., USA

\section{Glenda E Gray}

Office of the President, South African Medical Research Council, Cape Town, South Africa

\footnotetext{
1. Centers for Disease Control and Prevention. Wastewater surveillance: A new public health tool to understand COVID-19 spread in a community. 2021. https://www cdc gov/coronavirus/2019-ncov/ cases-updates/wastewater-surveillance.html (accessed 2 March 2021).

2. World Health Organization. COVID-19 strategic preparedness and response (SPRP): Monitoring and evaluation framework. 5 June 2020. https://www.who.int/publications/ $/$ /item/monitoring-andand evaluation framework. 5 June 2020 . https

3. World Health Organization Regional Office for Africa. Africa needs timely access to safe and effective COVID-19 vaccines. 21 January 2021. https://www.afro.who.int/news/africa-needs-timely-access-
Colitions COVID-19 vaccines. 21 January 2021. https://www.afro.wh)
safe-and-effective-covid-19-vaccines (accessed 2 March 2021).
}

4. Díaz-Pachón DA, Rao JS. A simple correction for covid-19 testing bias. J Theor Biol 2020;512(1):1-6. https://doi.org/10.1016/j.jtbi.2020.110556

Lipsitch M P il D Swerdlow D, Finelli L Defining the epidemiology of COVID-19 - studies needed. N Engl J Med 2020;382(13):1194-1196. https://doi.org/10.1056/NEJMp2002125

6. Singh J. COVID-19: Science and global health governance under attack. S Afr Med J 2020;110(6):445446. https://doi.org/10.7196/SAMJ.2020v110i5.14820

7. COVID-19 WBE Collaborative. COVID-19 poops dashboard. 2021. https://www.covid19wbec.org/ covidpoops19 (accessed 1 March 2021).

8. World Health Organization (Europe). Rapid expert consultation on environmental surveillance of SARSCoV-2 in wastewater. Summary report. Virtual meeting, 23 July 2020. Copenhagen: WHO Regional Office for Europe, 2020. https://apps.who.int/iris/handle/10665/334305 (accessed 5 March 2021).

9. Johnson R, Muller CJF, Ghoor S, et al. Qualitative and quantitative detection of SARS-CoV-2 RNA in untreated wastewater in Western Cape Province, South Africa. S Afr Med J 2021;111(3);198-202. https://doi.org/10.7196/SAMJ.2021.v111i3.15154

10. Hodgson S, Mansatta K, Mallett G, Harris V, Emary K, Pollard A. What defines an efficacious COVID-19 vaccine? A review of the challenges assessing the clinical efficacy of vaccines against SARSCoV-2. Lancet Infect Dis 2021;21(2):E26-E35. https://doi.org/10.1016/S1473-3099(20)30773-8

11. Smith T, Cassell G, Bhatnagar A. Wastewater surveillance can have a second act in COVID-19 vaccine distribution. JAMA Health Forum 2021;2(1). https:// doi.org/10.1001/jamahealthforum.2020.1616
dact

12. Farkas K, Hillary L, Malham S, McDonald J, Jones D. Wastewater and public health: The potential of wastewater surveillance for monitoring COVID-19. Curr Opin Environ Sci Health 2020;17(1):14-20. https://doi.org/10.1016/j.coesh.2020.06.00

S Afr Med J 2021;111(6):524-525. https://doi.org/10.7196/SAMJ.2021.v111i6.15698 